A N N A L E S Annales de Bretagne et des Pays de l'Ouest

\title{
La revendication bretonne du trône de France
} (1213-1358)

\section{Michael Jones}

\section{(2) OpenEdition \\ 1 Journals}

Édition électronique

URL : http://journals.openedition.org/abpo/3455

DOI : $10.4000 / a b p o .3455$

ISBN : 978-2-7535-5365-1

ISSN : 2108-6443

Éditeur

Presses universitaires de Rennes

Édition imprimée

Date de publication : 30 décembre 2016

Pagination : 193-196

ISBN : 978-2-7535-5363-7

ISSN : 0399-0826

\section{Référence électronique}

Michael Jones, «La revendication bretonne du trône de France (1213-1358) », Annales de Bretagne et des Pays de l'Ouest [En ligne], 123-4 | 2016, mis en ligne le 30 décembre 2016, consulté le 23 septembre 2020. URL : http://journals.openedition.org/abpo/3455 ; DOI : https://doi.org/10.4000/ abpo.3455 
héroïque irlandais Táin Bó Cuailnge. Comme d'autres régions des marges (Écosse, Bretagne, Finlande et Estonie), l'Irlande a érigé une histoire ancienne en "national epic of the Irish people ", à ceci près qu'il s'agit bien d'une œuvre médiévale puisque les copies les plus anciennes remontent au XII ${ }^{\mathrm{e}}$ siècle.

L'ouvrage se clôt sur les "marges du parchemin ». À partir des sceaux, Vincent Launay expose les choix opérés par les sires de Rais dont la seigneurie se situait à la marge entre Bretagne et Poitou. Après avoir eu recours au sceau du sénéchal de Nantes jusque dans les années 1270 , ils se tournèrent ensuite vers l'autorité royale, plus éloignée, garantissant ainsi une plus grande marge de liberté. Suivent trois contributions sur les marges des manuscrits dont plusieurs exemples sont reproduits en couleurs éclairant les observations des auteurs. Les dessins ou lettres (marginalia) des manuscrits gallois médiévaux sont présentés par Ceridwen LloydMorgan, si dans certains cas les marginalia commentent, illustrent ou aident à comprendre le texte, parfois ils paraissent être un simple divertissement tant pour l'exécutant que pour l'éventuel lecteur. En effet, dans Peniarth 23, fo $57 \mathrm{v}^{0}$ l'auteur émet l'hypothèse que les dessins réalisés en marge l'ont été ici car le mécène du manuscrit ne consultait vraisemblablement que les premières pages. À l'inverse et dans un autre type de manuscrit, Mégumi Tanabé montre que les ornements marginaux des Heures de Pierre II (1455-1457) et ceux des Heures de Marguerite d'Orléans (avant 1435) entrent en écho " avec les aspirations profondes du commanditaire". L'iconographie du manuscrit du duc breton est truffée d'allusions politiques, Pierre II reprend les codes royaux ou bien rappelle son soutien au mariage de sa nièce Marguerite avec son cousin François de Bretagne, union qui permit d'éviter une crise de succession car Pierre n'eut pas d'enfant. Le Missel Pontifical de Michel Guibé, évêque de Rennes (1482-1502), permet à Sophie Cassagnes-Brouquet de rappeler la place du commanditaire. Ses armoiries et la crosse épiscopale sont omniprésentes dans les décors marginaux. Et si parfois des scènes fantaisistes et des monstres surgissent il ne faut pas en conclure à une liberté créative et débridée des trois enlumineurs sans lien avec le lecteur, au contraire, tout comme les sculptures en bois et en pierre des cathédrales ces décors étaient " consubstantiel[s] " au christianisme médiéval, ils devaient conduire à une réflexion, une méditation.

Il s'agit donc d'un volume riche, dense et varié. Une question notamment le parcourt : celle de l'identité. Galvaudée depuis quelques années, elle mérite justement d'être revue. Si des études ont été menées il y a maintenant trente ans sur le "bretonnisme " (Jean-Yves Guiomar), la question vaut d'être reprise dans son ensemble à la lumière de travaux récents dont une partie des auteurs de ce volume sont précisément les contributeurs. Enfin, ce recueil fait mentir Lucien Febvre lorsque ce dernier estimait que " peu importe le cadre, la marge. C'est le cœur qui vaut, et qu'il faut avant tout considérer".

Julien BACHELIER

Copy, Jean-Yves, La revendication bretonne du trône de France (1213-1358), Paris, Alain Baudry et Cie, 2016, 310 p.

Il y a trente ans dans son livre Art, société et politique au temps des ducs de Bretagne. Les gisants haut-bretons (Paris, Aux Amateurs des Livres, 308 p.), JeanYves Copy publiait une étude révolutionnaire sur les gisants médiévaux; cette étude se fondait sur un inventaire de 272 monuments, tant disparus que conservés, du début du XIII ${ }^{\mathrm{e}}$ siècle à 1514, répertoriés principalement dans les trois départements 
des Côtes-du-Nord (maintenant Côtes-d'Armor), Ille-et-Vilaine et Loire-Atlantique. Il essayait quand cela était possible, à partir des aspects stylistiques et des changements dans la mode de la sculpture, de déterminer les influences que d'autres monuments dans d'autres régions avaient pu avoir sur les familles commanditaires ou sur la production des artisans de ce riche corpus de monuments. Mais cela mis à part, l'un des aspects les plus provocateurs de la synthèse de Copy était qu'il démontrait que derrière beaucoup de ces tombeaux on trouvait des facteurs politiques et idéologiques qui avaient contribué à déterminer leur forme ou leur symbolisme. Pour prendre un seul exemple, Copy a été le premier à porter une attention sérieuse, dans l'abbaye de Saint-Gildas-de-Rhuys, à l'apparition sur une pierre tombale d'une couronne soutenue par deux anges volant au-dessus de la tête du gisant de Jeanne, fille aînée de Jean IV, duc de Bretagne, et de sa troisième femme, Jeanne de Navarre, et qui mourut encore enfant en 1388 alors qu'elle était la seule héritière du duché. Il a relié ce symbolisme aux prétentions émergentes de la dynastie des Montfort à la royauté et à la monarchie, question depuis longtemps importante dans l'évolution du duché à la fin du Moyen Âge et sur son idéologie.

Dans le présent ouvrage il revient juste sur deux des monuments qu'il avait brièvement examinés dans Art, société et politique, en appliquant la même méthode et en insistant à l'aide d'arguments de poids sur leur signification politique ainsi que sur leur nouveauté artistique. Ce sont en réalité deux exemples tout à fait extraordinaires de l'art funéraire médiéval, en métal semi-précieux (principalement en alliage de cuivre), d'une richesse d'éléments héraldiques qui a peu d'équivalent en Europe, a fortiori en France (Signalons à ce sujet qu'une précieuse synthèse vient de paraître : BADHAM, Sally et OOSTERWIJK, Sophie, " "Monumentum aere perennius?" Precious-metal effigial tomb monuments in Europe 1080-1430 ", Church Monuments, t. xxx, 2015, p. 7-105).

La mode des monuments funéraires en métal est apparue dans l'Allemagne de la fin du Xle siècle et a été introduite en France par la cour des comtes de Champagne avec le gisant élevé à la mémoire du comte Henri le Libéral (mort en 1180). Mais c'est le savoir-faire des émailleurs de Limoges qui encouragea l'ajout d'un décor héraldique qui atteignit son apogée dans les dernières années du XIII ${ }^{\mathrm{e}}$ siècle, avant de se raréfier dès le début du XIV ${ }^{\mathrm{e}}$ siècle. C'est aussi une catégorie de monuments qui a énormément souffert des déprédations et destructions des générations suivantes à cause de leur attrait esthétique mais aussi de la valeur intrinsèque du métal dont étaient faits ces gisants ainsi que les plaques décoratives, ornementations et autres détails qui les accompagnaient, ce qui explique qu'il en reste très peu actuellement dans l'ensemble de l'Europe occidentale.

Les deux tombeaux étudiés sont - ou plutôt étaient puisqu'ils ont tous les deux disparu à la Révolution - celui d'un cadet de la famille ducale, Robert, troisième fils de Jean I ${ }^{\text {er }}$ et de Blanche de Champagne, qui mourut en $1260 \mathrm{n}$. st. et fut enterré dans le couvent des Franciscains de Nantes, et un monument à la mémoire d'Alix de Thouars, duchesse de Bretagne sua jure, épouse de Pierre Mauclerc, décédée en 1221, et de leur fille Yolande de Bretagne, sœur de Jean I ${ }^{\text {er }}$ et épouse de Hugues XI de Lusignan, comte de la Marche, décédée en 1272 après un long veuvage et enterrée dans l'abbaye de Villeneuve, fondée par leur mère et grand-mère la duchesse Constance dans la commune des Sorinières au Pays de Retz. Les deux tombeaux sont maintenant connus principalement grâce aux descriptions qu'en a faites Dubuisson-Aubenay dans son Itinéraire de 1636 et à des dessins réalisés un peu plus tard dans ce siècle par l'antiquaire Roger de Gaignières qui en révéla la splendeur héraldique. Dans le cas du tombeau de Robert, sur un petit coffre de pierre "élevée de 2 piés sur terre, longue de viron cinq et large viron de 2 " (Dubuisson-Aubenay), ou approximativement $153 \times 64 \mathrm{~cm}$, se trouvait un petit gisant de bois " élevée 
encore prez d'un pié, couverte d'une lame de cuivre émaillée à fleurs " tandis que tout autour sur le dessus et la base du tombeau se voyaient des plaques comportant 32 blasons héraldiques identifiables " le tout émaillé de diverses couleurs ". Si on laisse de côté le fait que très probablement, d'après l'analyse artistique, la tête d'Alix provient d'une tombe antérieure, le double tombeau d'Alix et Yolande, sous la forme où il existait au XVII ${ }^{\mathrm{e}}$ siècle, " longue de 6 piés ou environ et large de 4 à 5 , élevée sur terre de deux piés ", présentait les gisants des deux femmes allongées l'une à côté de l'autre " couverte d'une couverture entière ou lame qui déborde sur les costés, de cuivre doré, émaillé de moresques, orné d'écussons de aultres alliances et seigneuries, comme Bretagne, Angleterre, Navarre, etc. tout autour ". Les deux gisants étaient aussi de " cuivre doré, gisantes et vestues, celle du costé gauche [i.e. Alix] coronée d'une coronne aulte, mais simple et unie, sans fleurons, et tenant en sa main droite un sceptre fleuronné ", tandis que des inscriptions révélaient l'identité des deux femmes. La particularité la plus remarquable et semble-t-il, sans équivalent, résidait dans le fait que pas moins de 357 écus décoraient le dessus et les côtés du monument.

C'est principalement à l'identification des familles représentées sur ce tombeau et sur celui de Robert de Bretagne et aux implications politiques de ces programmes artistiques que J.-Y. Copy consacre sa pénétrante analyse. Dans le cas de Robert, de précédents chercheurs avaient déjà attiré l'attention sur les blasons héraldiques qui faisaient allusion aux revendications de Jean $\mathrm{I}^{\mathrm{er}}$ et Blanche de Champagne sur le trône de Navarre : celui-ci avait été détenu entre 1234 et 1253 par le père de Blanche, Thibaut IV, comte de Champagne, mais le couple ducal avait été forcé en 1254 d'y renoncer en faveur du demi-frère de Blanche, Thibaut $\mathrm{V}$, né d'un mariage ultérieur. J.-Y. Copy ici installe ces prétentions dans une perspective beaucoup plus longue, celle des premiers membres de la famille de Dreux-Bretagne en quête d'une couronne, puisque la " revendication " première du titre remonte à l'époque où les droits de Robert, quatrième fils de Louis VI (1108-1137), à la succession de la couronne de France à la suite de la mort de ses frères aînés, semble avoir été ignorés en faveur de ceux de son jeune frère le futur Louis VII. Ce fut fait au motif de la supposée incapacité de Robert et celui-ci fut plus tard dédommagé partiellement par l'attribution du comté de Dreux érigé en apanage. Pour J.-Y. Copy, cela explique pourquoi les descendants de Robert, parmi lesquels Pierre Mauclerc, son petit-fils, et Jean ${ }^{\text {er }}$, apparemment floués de leurs droits à la couronne capétienne gardaient des ambitions politiques royales. Cela explique aussi, avance-t-il, pourquoi ils investirent si massivement dans des entreprises architecturales et artistiques qui devaient à travers le langage des blasons et d'autres symboles informer les observateurs avertis de leur statut élevé et de leurs prétentions. De là s'ensuit une intéressante analyse sur le patronage des Dreux sur l'église collégiale de Saint-Yved de Braine (act. Aisne) qui devint la nécropole familiale, sur la rose du transept sud de la cathédrale de Chartres sur laquelle les armes de Pierre Mauclerc sont prééminentes (ce qui aurait prétendument provoqué une réponse royale dans la rose du transept nord) ainsi que sur les deux tombeaux précédemment décrits. Dans celui d'Alix et de Yolande de Dreux-Bretagne-Champagne l'héraldique est inextricablement mêlée à celle de la famille de Lusignan qui manœuvrait de son côté entre les couronnes d'Angleterre et de France de la fin du XII e siècle à la fin du XIII e siècle. Le domicile de ses membres en Angleterre au XIII ${ }^{\mathrm{e}}$ siècle, l'identification de nombreux autre écus anglais figurant sur le tombeau et les questions de la rivalité franco-anglaise dans les provinces voisines de la Gascogne, surtout après le traité de Paris (1259), forment ainsi une partie importante de l'étude de J.-Y. Copy.

Dans les limites d'un compte rendu, il est impossible de traiter aisément des nombreux fils que J.-Y. Copy a tissés en une riche synthèse subtile et imaginative 
à partir d'un important faisceau de témoignages, à la fois documentaires et matériels, pour soutenir sa thèse principale de " la revendication bretonne du trône de France " affichée dans l'héraldique des deux tombeaux. À cela il ajoute encore une dernière partie importante dans laquelle il argumente encore avec brio sur la façon dont les ambitions royales les plus anciennes des Dreux, ses prédécesseurs, ont laissé un héritage qui a influencé jusqu'à la manière dont le duc Charles de Blois (1341-1364) a choisi d'afficher ses propres ambitions monarchiques, même s'il s'appuya beaucoup plus sur les prétentions historiques plus lointaines des princes du duché à la royauté aux époques mérovingienne et carolingienne. Tous les lecteurs ne seront pas convaincus par tous ces arguments sur ces liens et sur la continuité d'une idéologie et des ambitions politiques de différentes générations à travers les siècles à une époque où la France et l'Angleterre médiévales elles-mêmes évoluaient rapidement. En particulier certains trouveront peut-être problématique l'association occasionnelle des noms " Plantagenêt " et "Anglais ", alors que, pour la plus grande partie de la période qui nous préoccupe, la famille des Plantagenêts était et se considérait elle-même, même après 1204 et la perte de la Normandie et de l'Anjou, comme faisant partie d'une France élargie plutôt que d'une Angleterre au sens étroit. Il y a aussi inévitablement un certain degré de spéculation sur les intentions de ceux qui ont commandité ces œuvres particulières, même des incertitudes quant à leur identité ainsi qu'à celle de certaines familles dont les armes sont représentées. Yolande de Bretagne, comtesse de la Marche, peut difficilement avoir agi seule en ordonnant le double tombeau de Villeneuve. Jusqu'où les autres membres de la famille de Lusignan (alors puissante en Angleterre) ou le duc Jean I $^{\text {er }}$ et ses conseillers sont-ils intervenus? Qui a proposé que la duchesse Alix soit représentée tenant l'attribut royal d'un sceptre? Mais ce que J.-Y. Copy a démontré sans ambigüité d'une manière astucieuse, passionnante et stimulante, c'est que les relations entre les dynasties régnantes de Bretagne et de France dans le dernier siècle du règne des Capétiens et sous les premiers Valois sont encore plus compliquées qu'on ne le savait auparavant et que l'histoire de l'art peut aider à les éclairer d'une manière plus spectaculaire que beaucoup d'entre nous pouvaient le penser.

Michael JONES

FAuCHERRE, Nicolas, GuillouËT, Jean-Marie (dir.), Nantes flamboyante, 1380-1530, Nantes, Société archéologique et historique de Nantes et de la Loire-Atlantique, 2014, $282 \mathrm{p}$.

Ce livre regroupe les contributions du colloque organisé en novembre 2011 par Nicolas Faucherre et Jean-Marie Guillouët à l'université de Nantes. Il est constitué de vingt-deux études qui se concentrent sur les biens matériels, architecturaux et décoratifs datant de cette période de transition entre le Moyen Âge et les Temps modernes, articulées à l'histoire politique d'un espace urbain et provincial en mutation et en essor. L'ambition de cet ouvrage collectif est de définir la " forme de la ville " dans sa complexité entre 1380 et 1530, période charnière de l'histoire de l'architecture, entre gothique et Renaissance. Une douzaine de lieux parfaitement situés et disséqués dans leur intimité architecturale sont étudiés sur le temps long, donnant au lecteur une image très nette de ce que fut un dispositif topographique nantais qui a aujourd'hui presque intégralement disparu. Sa restitution relève, à bien des égards, de la performance archéologique et historique. 